\title{
IncRNA PCAT6 facilitates cell proliferation and invasion via regulating the miR-326/hnRNPA2B1 axis in liver cancer
}

\author{
JUN LUO $^{1 *}$, JIAPING ZHENG ${ }^{2 *}$, WEIYUAN HAO $^{2}$, HUI ZENG $^{2}$, ZHEWEI ZHANG $^{2}$ and GUOLIANG SHAO ${ }^{2}$ \\ ${ }^{1}$ The Second Clinical Medical College, Zhejiang Chinese Medical University; ${ }^{2}$ Department of Intervention, \\ The Cancer Hospital of the University of Chinese Academy of Sciences, Zhejiang Cancer Hospital, \\ Institute of Basic Medicine and Cancer, Chinese Academy of Sciences, Hangzhou, Zhejiang 310022, P.R. China
}

Received September 17, 2020; Accepted February 26, 2021

DOI: $10.3892 / \mathrm{ol} .2021 .12732$

\begin{abstract}
Liver cancer is one of the most common malignant human tumors with the highest morbidity and mortality rates of all cancer types in China. Evidence suggests that long non-coding RNA prostate cancer-associated transcript 6 (PCAT6) plays an essential role in tumor progression. However, the roles and mechanism of PCAT6 in liver cancer remain unclear. The present study showed that the expression of PCAT6 and heterogeneous nuclear ribonucleoprotein A2B1 (hnRNPA2B1) was upregulated in liver cancer tissues compared with non-cancerous tissues and were associated with poor overall survival time, whereas microRNA (miR)-326 expression was downregulated. Moreover, knockdown of PCAT6 significantly inhibited the proliferation and invasion of liver cancer cells in vitro and in vivo. A dual-luciferase reporter gene assay demonstrated that PCAT6 could bind to miR-326 and that hnRNPA2B1 was a direct target gene of miR-326. Mechanistically, silenced PCAT6 suppressed the malignant phenotype of liver cancer cells through upregulating the inhibitory effect of miR-326 on hnRNPA2B1 expression. Taken together, these data demonstrated that knockdown of PCAT6 inhibited liver cancer progression through regulation
\end{abstract}

Correspondence to: Dr Guoliang Shao, Department of Intervention, The Cancer Hospital of the University of Chinese Academy of Sciences, Zhejiang Cancer Hospital, Institute of Basic Medicine and Cancer, Chinese Academy of Sciences, 1 East Banshan Road, Hangzhou, Zhejiang 310022, P.R. China

E-mail: glshao123@163.com

Abbreviations: ANT, adjacent normal tissue; CCK-8, Cell Counting Kit-8; GEPIA, Gene Expression Profiling Interactive Analysis; HCCT, liver cancer tissue; HCC, hepatocellular carcinoma; hnRNPA2B1, heterogeneous nuclear ribonucleoprotein A2B1; PCAT6, prostate cancer-associated transcript 6; MUT, mutant type; RT-qPCR, reverse transcription-quantitative polymerase chain reaction; TCGA, The Cancer Genome Atlas; WT, wild-type

${ }^{*}$ Contributed equally

Key words: liver cancer, lncRNA PCAT6, miRNA-326, hnRNPA2B1 of the miR-326/hnRNPA2B1 axis, suggesting that PCAT6 functions as an oncogene and may be a useful biomarker for the future diagnosis and treatment of liver cancer.

\section{Introduction}

Liver cancer is one of the most common types of cancer and is a serious threat to human health, with 854,000 new cases of liver cancer and 810,000 associated deaths globally in 2015, contributing to 20,578,000 disability-adjusted life-years (1). After surgery, the incidence of tumor recurrence and metastasis was high from 1990-2012 globally, at 33-100\% (2) and the prognosis is poor (3). In recent years, surgical techniques have developed with the improvement of diagnostic methods. However, the clinical outcome of patients with unresectable disease is suboptimal with a median survival of only 12 months and no patient survived beyond 5 years from 1986-2003 in the USA $(4,5)$. Therefore, it is important to identify novel therapeutic targets and improve the diagnosis and treatment of patients with liver cancer.

Long non-coding RNA (lncRNA) is an RNA molecule with $>200$ nucleotides and lacking protein-coding potential. Research has implicated lncRNAs may function as oncogene or tumor suppressor genes by regulating the transcription of protein-coding genes or altering chromatin structure $(6,7)$. Moreover, lncRNAs have been recognized as diagnostic molecular biomarkers or therapeutic targets of cancer $(8,9)$. For example, IncRNA H19, SNHG11 and PTCSC3 are abnormally expressed in tumor tissues and cell lines, and can serve as biomarkers for the diagnosis and prognosis of numerous solid tumors, including breast (10), colorectal (11) and gastric cancer (12). IncRNAs are involved in the development and progression of malignant tumors by regulating cancer cell proliferation, migration, invasion, apoptosis, angiogenesis and immune escape (13). Previously, prostate cancer-associated transcript 6 (PCAT6) has been identified as an lncRNA involved in the regulation of lung cancer progression (14). Subsequently, several studies have highlighted the abnormal expression of lncRNA PCAT6 in various malignant tumors, including gastrointestinal stromal tumor, colon cancer and osteosarcoma, suggesting that PCAT6 may play an essential role in tumor progression (15-17). Although the oncogenic roles of PCAT6 have been demonstrated in several tumors, 
little is known about the functionality and mechanism of PCAT6 in liver cancer. Moreover, evidence has shown that the exerted actions of micro (mi)RNAs depend on the modulation of upstream molecules, including lncRNAs, and downstream mRNAs (18). Evidence has suggested that microRNA (miRNA/miR) expression is abnormal in different types of tumor tissues and it is well-known that miRNAs play notable roles in tumor development and cancer therapy (19). To date, low expression levels of miR-326 have been observed in HCC tissues and cell lines, and miR-326 has been found to exert a regulatory effect on the proliferation, migration and invasion of HCC cells (20). Nevertheless, to the best of the authors' knowledge, there is currently no evidence regarding the interaction between PCAT6 and miR-326 in liver cancer.

In the present study, based on the Gene Expression Profiling Interactive Analysis (GEPIA) database, the expression of IncRNA PCAT6 between liver cancer tissues and adjacent noncancerous liver tissues was analyzed. Furthermore, the functional role and mechanism of PCAT6 was explored by performing a series of experiments in vitro and in vivo. Taken together, therapeutic interventions targeting PCAT6 could be developed as a new strategy for the treatment of liver cancer.

\section{Materials and methods}

Patients and specimens. All tissue specimens (ANT, adjacent normal tissues; HCCT, liver cancer tissues) were collected from 117 patients with liver cancer, which were histologically confirmed by an experienced pathologist (GS) at The Department of Herpetological Surgery, Zhejiang Cancer Hospital (Hangzhou, China) between January 2015 and January 2017. The age range of 117 patients was 45-80 years (mean age, 58 years old), 68 were females and 49 were males. The inclusion criteria were as follows: i) $\geq 18$ Years old; ii) histologically diagnosed with liver cancer and were amenable to surgery; iii) had never undergone any type of anti-cancer therapy, such as chemotherapy and radiotherapy, before surgery; iv) clinical data were complete. The exclusion criteria were as follows: Patients who also had other i) malignant tumors; ii) severe cardiovascular and cerebrovascular diseases and iii) hematological diseases. Tissue specimens were immediately kept in RNA Keeper Tissue Stabilizer (Vazyme Biotech Co., Ltd.) after surgical resection and then stored at $-80^{\circ} \mathrm{C}$. The study protocol was reviewed and approved by The Ethics Board of Zhejiang Cancer Hospital (Hangzhou, China). The study was conducted as per the principles stated in the Declaration of Helsinki and local ethical guidance documents (21). Written informed consent was obtained from all patients from whom specimens were collected.

GEPIA database. GEPIA (http://gepia.cancer-pku.cn/) is a database that provides key interactive and customizable functions, including differential expression analysis, and patient survival analysis (22). Following submission of an analysis request, GEPIA can provide the visual image results for users. The gene symbol PCAT6 and hnRNPA2B1 were submitted on GEPIA for differential expression analysis between data from liver cancer tissues and adjacent normal tissues, and provided results consisting of box plots and survival analysis. A total of 364 cases of liver cancer patients were divided into groups according to the median expression of PCAT6 or hnRNPA2B1: A high PCAT6 or hnRNPA2B1 expression group and low PCAT6 expression group. The median of relative PCAT6 expression level was 1.5, so the number of patients with high PCAT6 expression were 182 $(>1.5)$ and the number of patients with low PCAT6 expression were $182(\leq 1.5)$. The median of relative hnRNPA2B1 expression level was 8.2, so the number of patients with high hnRNPA2B1 expression were $182(>8.2)$ and the number of patients with low hnRNPA2B1 expression were $182(\leq 8.2)$.

Cell culture and transfection. Human liver cancer cell lines MHCC97H, HepG2 and Huh7 and the normal liver epithelial cells THLE-3 and 293 T cells were obtained from The American Type Culture Collection and cultured according to their recommendations. The liver cancer cell lines were authenticated by short tandem repeat profiling.

PCAT6 short hairpin (sh)RNA and shRNA scrambled control (sh-NC), pcDNA3.1-PCAT6 and empty vector (pcDNA3.1-NC), miR-326 mimic and a scrambled negative control (NC mimic), miR-326 inhibitor (miR-Inh) and a scrambled negative control (miR-NC), hnRNPA2B1 shRNA and scrambled control (sh-NC) were purchased from GenePharm Co., Ltd. Both HepG2 and Huh7 cells were seeded into seeded in six-well plates $\left(2 \times 10^{5}\right.$ cells/well $)$ and then transfected with an oligonucleotide (50 nM miRNA mimic/inhibitor) or plasmid $(2 \mu \mathrm{g})$ using Lipofectamine ${ }^{\circledR}$ 3000 reagent (Thermo Fisher Scientific, Inc.) at $37^{\circ} \mathrm{C}$ for $24 \mathrm{~h}$. In vivo experiments, the lentiviral vectors carrying shRNA for PCAT6 (sh-PCAT6) or negative control (sh-NC) were synthesized by RiboBio Co., Ltd.

Reverse transcription-quantitative polymerase chain reaction (RT-qPCR). Total RNA was extracted from liver cancer tissues, adjacent non-cancerous tissues, liver cancer cell lines and normal liver epithelial cells using TRIzol ${ }^{\circledR}$ reagent (Invitrogen; Thermo Fisher Scientific, Inc.) as described in a previous study (23). cDNA was synthesized from $1 \mu \mathrm{g}$ of total RNA using the PrimeScript ${ }^{\mathrm{TM}}$ 1st Strand cDNA synthesis kit (Takara Bio, Inc.) according to the manufacturer's instructions. qPCR was performed in an ABI 7500 instrument (Thermo Fisher Scientific, Inc.) using SYBR ${ }^{\circledR}$-Green Real-Time PCR Master Mix (Takara Bio, Inc.) following the manufacturer's protocols. The PCR reaction conditions were initial denaturation at $95^{\circ} \mathrm{C}$ for $10 \mathrm{~min}$, followed by 40 cycles of $95^{\circ} \mathrm{C}$ for $15 \mathrm{sec}$ and $60^{\circ} \mathrm{C}$ for $30 \mathrm{sec}$. The following primer sequences were used for qPCR: PCAT6, Forward: 5'-CAGGAACCCCCTCCTTACTC-3' and reverse: 5'-CTAGGGATGTGTCCGAAGGA-3'; miR-326, forward: 5'-GGCGCCCAGAUAAUGCG-3', reverse: 5'-CGT GCAGGGTCCGAGGTC-3'; hnRNPA2B1, forward: 5'-GCT GTAGCAAGAGAGGAATCTGGA-3' and reverse: 5'-GCT TCTTCACAGTTACATGAGCCC-3'; GAPDH, forward: 5'-AGCCACATCGCTCAGACAC-3' and reverse: 5'-GCCCAA TACGACCAAATCC-3'; U6, forward: 5'-CTCGCTTCGGCA GCACA-3' and reverse: 5'-AACGCTTCACGAATTTGCGT-3'. The expression levels of PCAT6, hnRNPA2B1 and miR-326 were calculated using the $2^{-\Delta \Delta \mathrm{Cq}}$ method (24) and normalized to GAPDH and U6 as appropriate.

Cell counting kit-8(CCK-8) assay. After 24-h post-transfection, both HepG2 and Huh7 cells $\left(2 \times 10^{3}\right.$ cells/well) were seeded in 
96-well plates and cultured in DMEM medium (Gibco; Thermo Fisher Scientific, Inc.) supplemented with 10\% FBS (Gibco; Thermo Fisher Scientific, Inc.). When the density of the cells reached $70 \%$, cells were transfected with PCAT6 shRNA and sh-NC, miR-326 mimic and NC mimic, miR-326 inhibitor and inhibitor NC, or hnRNPA2B1 shRNA and vector. After $48 \mathrm{~h}$ of transfection, $10 \mu \mathrm{l}$ CCK-8 solution (Beyotime Institute of Biotechnology) was added to each well. After a 2-h incubation at $37^{\circ} \mathrm{C}$, the absorbance of each well was assessed at $450 \mathrm{~nm}$ using a microplate reader (Thermo Fisher Scientific, Inc.).

Colony formation assay. Both HepG2 and Huh7 cells were transfected with sh-hnRNPA2B1 and sh-NC, sh-PCAT6 and sh-NC, miR-326 inhibitor and inhibitor NC, pcDNA3.1-PCAT6 and (pcDNA3.1-NC), and cultured under standard conditions. After that, the cells were diluted and seeded into six-well plates at the density of 3,000 cells per well for 14 days. The cells were then stained with $0.5 \%$ crystal violet (Beyotime Institute of Biotechnology) at $37^{\circ} \mathrm{C}$ for $45 \mathrm{~min}$. The colony number was observed with an inverted microscope (magnification, $\mathrm{x} 10$; Thermo Fisher Scientific, Inc.) and counted using ImageJ software (version 4.0; National Institutes of Health).

Western blotting. To extract total protein, both HepG2 and Huh7 cells were lysed with RIPA Lysis Buffer (Beyotime Institute of Biotechnology) as described previously (25). The lysate was centrifuged at $1,200 \mathrm{xg}$ at $4^{\circ} \mathrm{C}$ for $10 \mathrm{~min}$. The protein amount in the supernatant was quantified using a BCA assay. Subsequently, protein specimens (40 $\mu \mathrm{g} / \mathrm{lane}$ ) were added onto SDS-PAGE, and electrophoresis at 60 V.Proteins were then transferred to a polyvinylidene difluoride membrane (EMD Millipore). After blocking with $5 \%(\mathrm{w} / \mathrm{v})$ non-fat dry milk at $37^{\circ} \mathrm{C}$ for $1 \mathrm{~h}$, the membranes were incubated with primary antibodies against $\beta$-actin $(1: 2,000$ dilution; cat. no. ab8226) and hnRNPA2B1 (1:1,000 dilution; cat. no. ab31645) at $4^{\circ} \mathrm{C}$ overnight. All antibodies were purchased from Abcam. Next, HRP-conjugated secondary antibodies (1:5,000 dilution; cat. no. HRP-60008, ProteinTech Group, Inc.) were added and incubated for $1 \mathrm{~h}$ at room temperature. The protein bands were detected using chemiluminescence (Pierce; Thermo Fisher Scientific, Inc.) and visualized on a Tanon 5200 Imaging system (Tanon Science \& Technology Co., Ltd.).

Transwell chamber assay. A Transwell chamber $(8-\mu \mathrm{m}$ pore, 24-well plate; Corning, Inc.) with insert membranes coated with diluted Matrigel at $37^{\circ} \mathrm{C}$ for $1 \mathrm{~h}$ was used. HepG2 or Huh7 cells at 24 -h post-transfection in serum-free medium $\left(1 \times 10^{5}\right.$ cells $)$ were added to the upper chamber and cultured at $37^{\circ} \mathrm{C}$ for $24 \mathrm{~h}$. The bottom chamber contained a medium with $10 \%$ FBS. After incubation at $37^{\circ} \mathrm{C}$ for $24 \mathrm{~h}$, the insert membranes were cut and stained with crystal violet at $37^{\circ} \mathrm{C}$ for $30 \mathrm{~min}$ (Beijing Solarbio Science \& Technology Co., Ltd.) and images were captured using an inverted microscope (magnification, x100). The number of invading cells were counted in three wells per group.

Bioinformatics. Starbase version 2.0 (http://starbase.sysu.edu. cn/index.php) (26) were browsed to identify the miRNAs that bind lncRNA PCAT6 and hnRNPA2B1.

Dual-luciferase reporter gene assay. The wild-type (WT) sequence of PCAT6 or the hnRNPA2B1 3' untranslated region
(UTR) containing binding sites of miR-326 was cloned into pmirGLO (Promega Corporation) to form the corresponding luciferase reporter vectors (WT-PCAT6 and hnRNPA2B1 3'UTR-WT). The mutant (MUT)-PCAT6 and hnRNPA2B1 3'UTR-MUT were obtained by mutating the seed sites. 293T cells were seeded into 24 -well plates at $1 \times 10^{5}$ cells $/ \mathrm{cm}^{2}$. When the cell density reached $70-80 \%$, reporter gene plasmids and miR-326 or control vector (GenePharm Co., Ltd.) were co-transfected into $293 \mathrm{~T}$ cells using Lipofectamine ${ }^{\circledR} 3000$ (Thermo Fisher Scientific, Inc.) at $37^{\circ} \mathrm{C}$ for $48 \mathrm{~h}$. The relative luciferase activities were detected using the Dual-Luciferase Reporter assay kit (Promega Corporation) according to the manufacturer's protocol. Luciferase activity was measured and normalized to Renilla luciferase activity. The sequences of miRNA mimic/inhibitor were as follows: miR-NC, 5'-UUC UCCGAACGUUCACGUTT-3'; miR-326 mimics, 5'-AGG AUGUCUAAAUGUUUGUUA-3' and miR-326 inhibitor, 5'-AAGAAGUGCACCAUGUUUGUUU-3'.

Xenograft model. A total of $20 \mathrm{BALB} / \mathrm{c}$ nude mice (male; 5-weeks-old, 20-22 g) were obtained from the Hunan SJA Laboratory Animal Co., Ltd. All mice had access to food and water ad libitum, and temperature in the cages was maintained at $24 \pm 1^{\circ} \mathrm{C}$ with a $12 / 12 \mathrm{~h}$ light/dark cycle and $40-50 \%$ humidity. Transfected HepG2 cells $\left(1 \times 10^{7}\right)$ were resuspended in $100 \mu \mathrm{l}$ PBS and subcutaneously injected into the flanks of male BALB/c mice $(n=10)$ as previously described (27). Tumor volume was detected every 4 days and calculated as: Length $(\mathrm{mm}) \mathrm{x}$ width ${ }^{2}\left(\mathrm{~mm}^{2}\right) / 2$. After tumor growth for 28 days, mice (weight, 21-24 g) were euthanized via cervical dislocation. Tumor samples were weighed and harvested to examine the expression of miR-326 and hnRNPA2B1. The animal experiments were conducted under the approval of The Animal Care and Use Committee of Zhejiang Cancer Hospital (approval no. IRB-2019-5).

Statistical analysis. All experiments were repeated at least three times. Data are presented as the mean \pm standard deviation (unless otherwise shown). The median expression of PCAT6 and hnRNPA2B1 in all human liver cancer samples were considered as the cut-off to classify groups into PCAT6and hnRNPA2B1-high and PCAT6-and hnRNPA2B1-low expression. Statistical analysis was performed using GraphPad Prism 8 (GraphPad Software, Inc.), and differences between groups were analyzed using one-way ANOVA and Tukey's post hoc tests. A paired or unpaired two-tailed Student's t-test was used to compare differences between two groups of paired tissues or unpaired cells, respectively. The correlation relationships among PCAT6, miR-326 and hnRNPA2B1 were analyzed using the Pearson's correlation coefficient. Survival analysis was performed using log-rank tests and presented on Kaplan-Meier plots. Survival curves with cross-over were compared using the Renyi test. $\mathrm{P}<0.05$ was considered to indicate a statistically significant difference.

\section{Results}

PCAT6 is upregulated and associated with the progression of liver cancer. The effect of PCAT6 on the progression of liver cancer was investigated using the GEPIA database. It was 

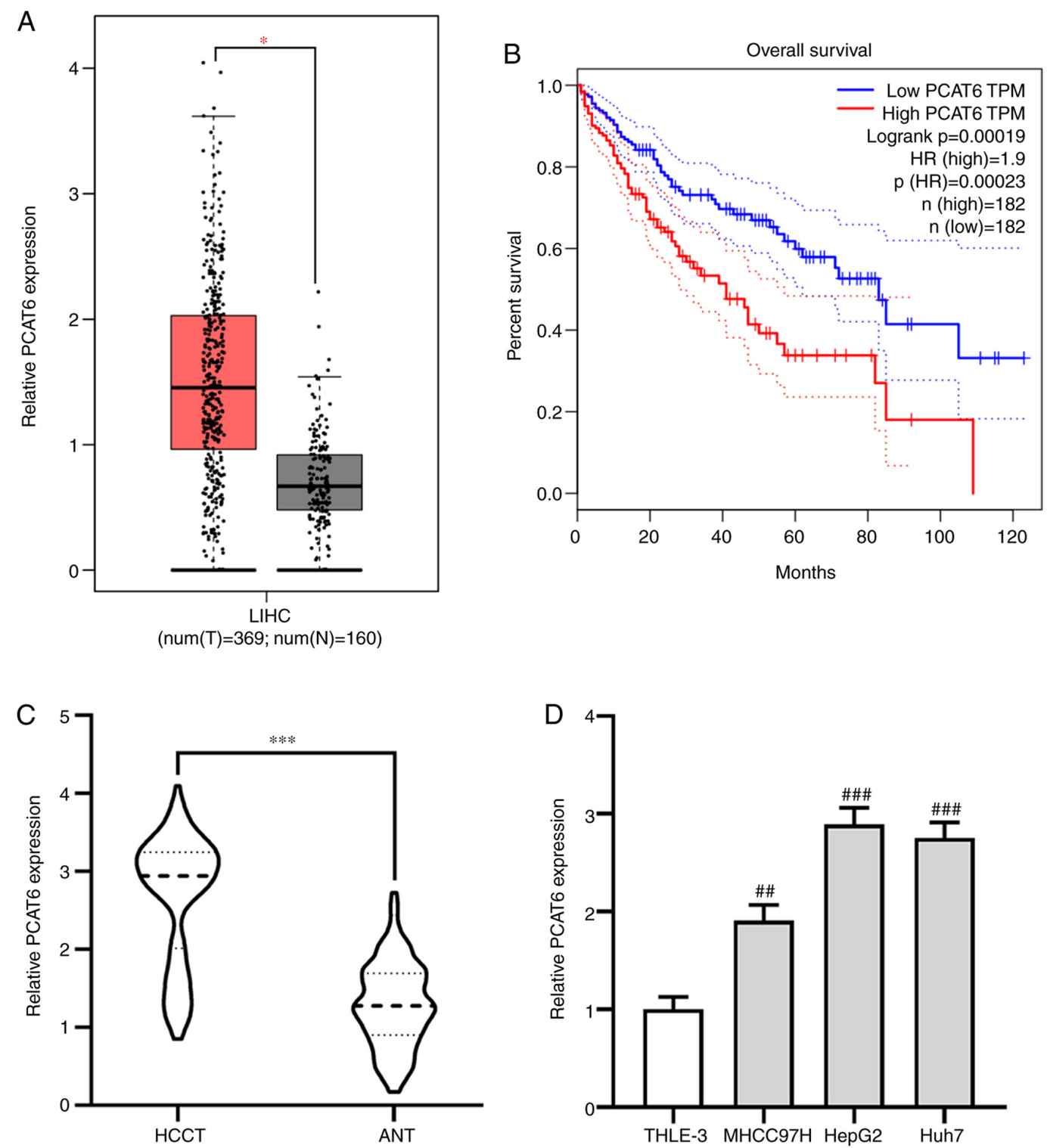

Figure 1. PCAT6 is upregulated in liver cancer tissues and associated with clinicopathological characteristics of patients with liver cancer. (A) Expression of PCAT6 in liver cancer tissues $(\mathrm{n}=369)$ and normal tissues $(\mathrm{n}=160)$ from the GEPIA database. (B) Association between PCAT6 expression and overall survival rate of patients with liver cancer was analyzed using GEPIA database. (C) RT-qPCR was performed to examine the expression of PCAT6 in HCCT (n=117) and matched adjacent normal tissues. (D) RT-qPCR was used to detect the expression of PCAT6 in liver cancer cell lines (MHCC97H, HepG2 and Huh7) and normal liver epithelial cells THLE-3. ${ }^{*} \mathrm{P}<0.05$ and ${ }^{* * * *} \mathrm{P}<0.001$ compared with the normal tissues; ${ }^{* \#} \mathrm{P}<0.01$ and ${ }^{\# \# \#} \mathrm{P}<0.001$ compared with the THLE-3 cell line. PCAT6, prostate cancer-associated transcript 6; GEPIA, Gene Expression Profiling Interactive Analysis; RT-qPCR, reverse transcription-quantitative PCR; HCCT, liver cancer tissue; ANT, adjacent normal tissues; LIHC, liver hepatocellular carcinoma; TPM, transcripts per million; HR, hazard ratio.

found that the expression of PCAT6 was significantly elevated in liver cancer tissues (HCCT) compared with adjacent normal tissues (ANT; P<0.05; Fig. 1A). Moreover, patients with liver cancer who had a high level of PCAT6 exhibited a shorter overall survival $(\mathrm{P}<0.001$; Fig. 1B). The expression pattern of PCAT6 in 117 pairs of HCCT and matching ANT was further investigated using RT-qPCR. It was found that PCAT6 expression was upregulated in HCCT compared with the ANT ( $\mathrm{P}<0.001$; Fig. 1C).Additionally, PCAT6 expressions in liver cancer cell lines MHCC97H, HepG2 and Huh7 were significantly enhanced compared with expression in THLE-3 normal liver epithelial cells, especially HepG2 and Huh7 cells (MHCC97H P<0.01; HepG2 and Huh7 P<0.001; Fig. 1D). Taken together, these data suggested that PCAT6 may act as an oncogene in the progression of liver cancer.
Knockdown of PCAT6 inhibits liver cancer cell proliferation and invasion. To investigate the biological functions of PCAT6 in liver cancer cells in vitro, sh-PCAT6 was transfected into both HepG2 and Huh7 cells to decrease its expression $(\mathrm{P}<0.001$; Fig. 2A). The CCK-8 assay showed that knockdown of PCAT6 significantly inhibited liver cancer cell proliferation ability compared with the sh-NC group ( $\mathrm{P}<0.05$; Fig. $2 \mathrm{~B}$ and $\mathrm{C})$. Colony formation assays showed that PCAT6-knockdown markedly reduced the number of liver cancer cell colonies compared with the sh-NC group ( $\mathrm{P}<0.001$; Fig. 2D). Furthermore, inhibition of PCAT6 weakened the invasion capacity of both HepG2 and Huh7 cells $(\mathrm{P}<0.001$; Fig. 2E). These data indicated that knockdown of PCAT6 exhibited an antitumor function in liver cancer by suppressing the proliferation and invasion abilities of both HepG2 and Huh7 cells. 

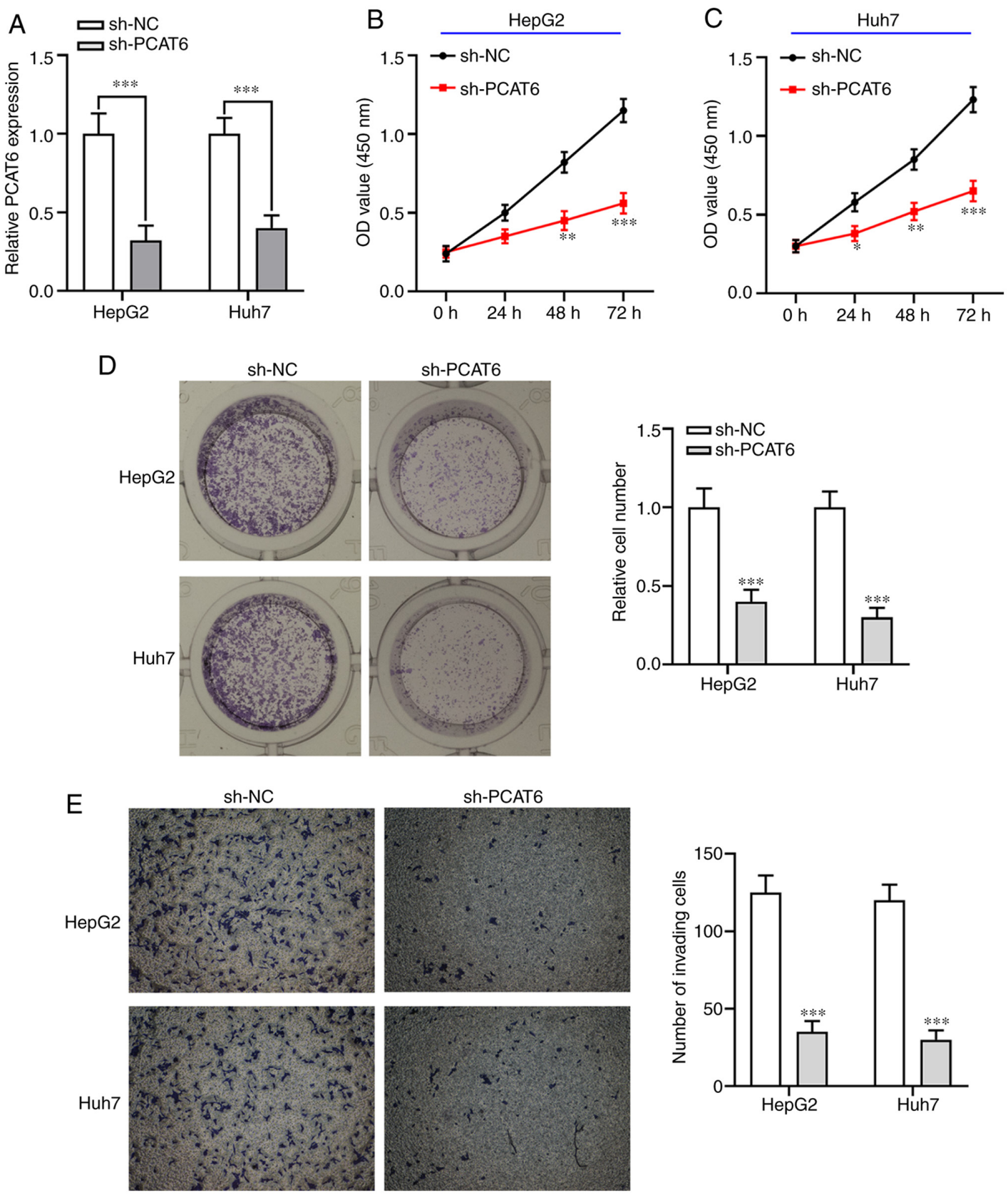

Figure 2. Knockdown of PCAT6 inhibits cell proliferation and invasion in liver cancer in vitro. (A) Both HepG2 and Huh7 cells were transfected with sh-PCAT6 or sh-NC and reverse transcription-quantitative PCR was used to detect the expression of PCAT6 in both cells. (B and C) Cell Counting Kit-8 assay was used to examine the cell proliferation in both HepG2 and Huh7 cell transfected with sh-PCAT6. (D) After transfection, colony formation of HepG2 and Huh7 was determined. (E) Transwell assay was applied to detect the invasive activity of liver cancer cells after transfection with sh-PCAT6 and sh-NC ${ }^{*} \mathrm{P}<0.05,{ }^{* *} \mathrm{P}<0.01$ and ${ }^{* * *} \mathrm{P}<0.001$ compared with sh-NC. PCAT6, prostate cancer-associated transcript 6; sh-, short hairpin-; NC, negative control.

miR-326 is a target gene of PCAT6. To further probe the underlying mechanism of PCAT6 function in liver cancer, the bioinformatics database, Starbase, was used to predict potential miRNAs that may be downstream target genes of PCAT6. Bioinformatics analysis showed that miR-326 could be a candidate target gene of PCAT6, as it has a potential binding site for PCAT6 (Fig. 3A). Notably, RT-qPCR revealed that the expression of miR-326 was lower in the HCCT group compared with that in the ANT group $(\mathrm{P}<0.001 ; \mathrm{Fig}$. 3B). A negative correlation was also found between miR-326 and PCAT6 in ANT group $(\mathrm{P}<0.001$; Fig. 3C). Subsequently, the luciferase reporter assay showed that overexpression of miR-326 significantly reduced the luciferase activity of
293T cells transfected with the WT-PCAT6 vector $(\mathrm{P}<0.001$; Fig. 3D), while the luciferase activity of the MUT-PCAT6 was not changed. Moreover, RT-qPCR showed that knockdown of PCAT6 markedly enhanced the expression of miR-326 in both HepG2 and Huh7 cells ( $\mathrm{P}<0.001$; Fig. 3E). Collectively, these results indicated that miR-326 was a target gene of PCAT6.

Inhibition of miR-326 abrogates the inhibitory effect of PCAT6-silencing on liver cancer cell proliferation and invasion. To further explore whether PCAT6 modulates the malignant phenotype of liver cancer cells by targeting miR-326, HepG2 and Huh7 cells were transfected with miR-Inh or miR-Inh + sh-PCAT6 $(\mathrm{P}<0.001$; Fig. 4A). The CCK-8 assay 
A

PCAT6-WT 5'-ggGGCCGGAGCUCCCAGAGg-3'
miR-326 3'-gaCCUCCUUCCCGGGUCUCe-5'

PCAT6-MUT 5'-ggGGCCGGAGUCGGGUCUCg-3'

D

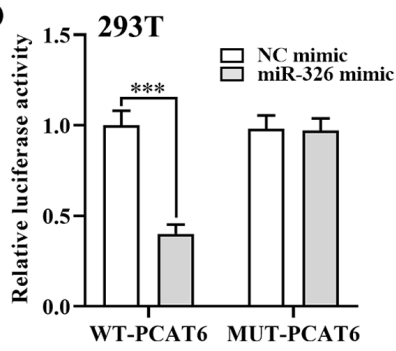

$\mathbf{F}$

hnRNPA2B1-WT 5'-auGGCAGAAGACACCAGAGc-3' \|\|$\|$ miR-326 3'-gaCCUCCUUCCCGGGUCUCc-5'

hnRNPA2B1-MUT 5'-auGGCAGAAGACAGGUCUCc-3'
B

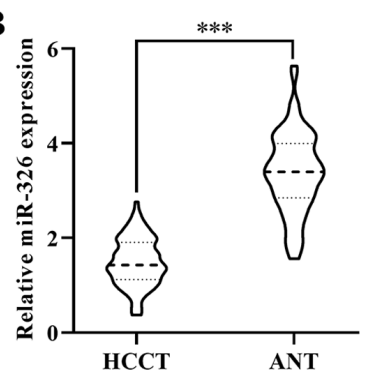

$\mathbf{E}$

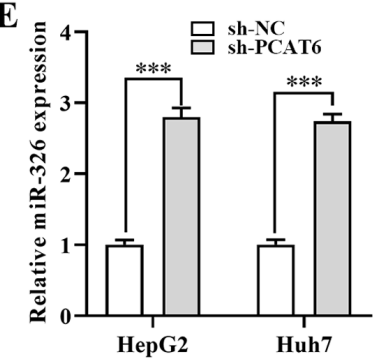

C

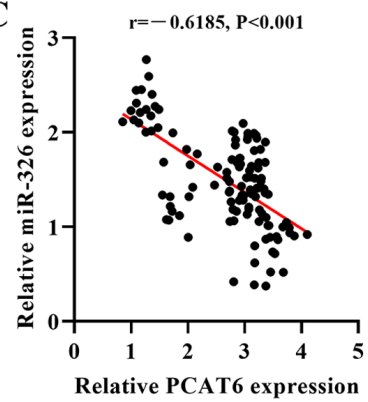

G
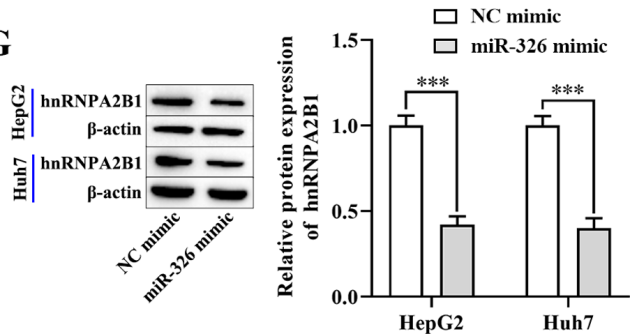

$\mathbf{J}$

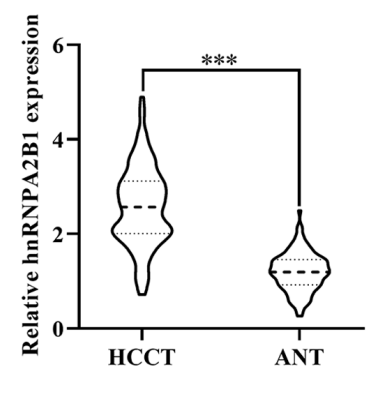

M

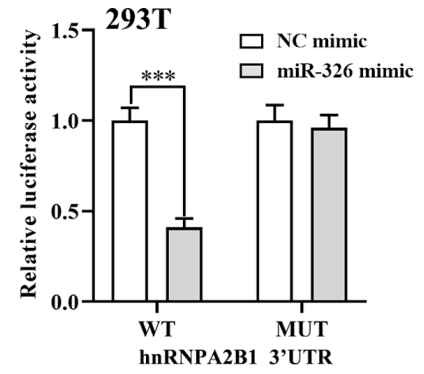

Figure 3. PCAT6 promotes hnRNPA2B1 expression in liver cancer cells by targeting miR-326. (A) Starbase predicted binding sites between miR-326 and PCAT6. (B) RT-qPCR detected the expression of miR-326 in HCCT ( $\mathrm{n}=117$ ) and matched ANT. (C) Pearson's correlation analysis between PCAT6 and miR-326 in liver cancer tissues. (D) Luciferase reporter assays were performed in 293T cells to detect the binding affinity of miR-326 to the PCAT6 3'UTR-WT or MUT. (E) Expression of miR-326 in both HepG2 and Huh7 cells after transfection with sh-PCAT6 or sh-NC examined using RT-qPCR. (F) Starbase database binding site predictions between miR-326 and hnRNPA2B1. (G) Protein levels of hnRNPA2B1 in both HepG2 and Huh7 cells after transfection with miR-326 mimic and NC mimic were examined using western blotting. (H) Expression of hnRNPA2B1 in liver cancer tissues $(\mathrm{n}=369)$ and normal tissues $(\mathrm{n}=160)$ from the GEPIA database. (I) Association between hnRNPA2B1 expression and overall survival rate of patients with liver cancer was analyzed using GEPIA database. (J) RT-qPCR detected the mRNA levels of hnRNPA2B1 in HCCT ( $\mathrm{n}=117)$ and matched ANT. (K and L) Correlation between hnRNPA2B1 and PCAT6 or miR-326 in liver cancer tissues. (M) Dual-luciferase reporter assay verified the negative regulatory association between miR-326 and hnRNPA2B1 in 293 T cells. ${ }^{*} \mathrm{P}<0.05$ and ${ }^{* * * *} \mathrm{P}<0.001$ compared with respective control. PCAT6, prostate cancer-associated transcript 6; hnRNPA2B1, heterogeneous nuclear ribonucleoprotein A2B1; GEPIA, Gene Expression Profiling Interactive Analysis; RT-qPCR, reverse transcription-quantitative PCR; HCCT, liver cancer tissue; ANT, adjacent normal tissue; miR-, microRNA; WT, wild-type; MUT, mutant; NC, negative control; HR, hazard ratio; LIHC; UTR, untranslated region. 
A

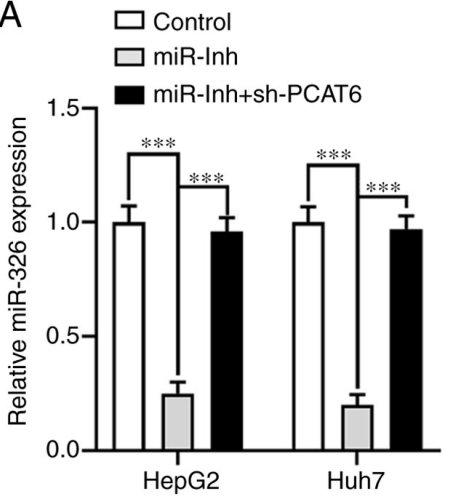

B

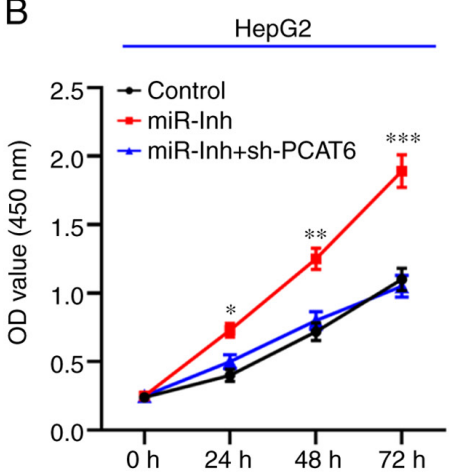

C

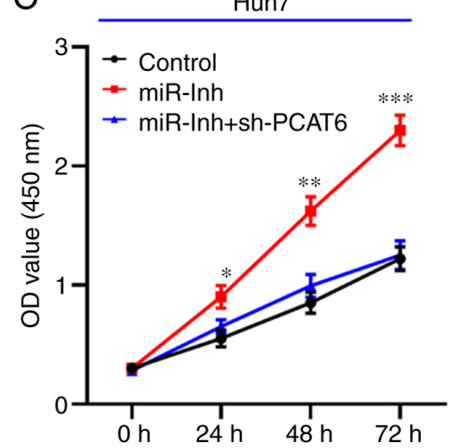

$\mathrm{D}$
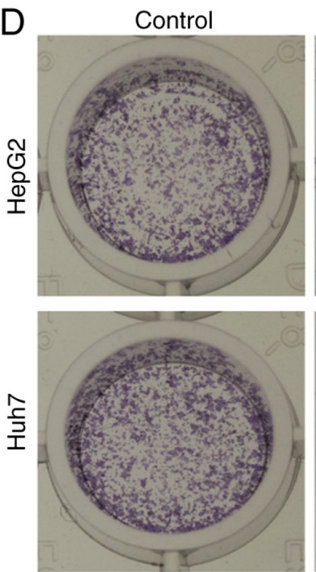

E
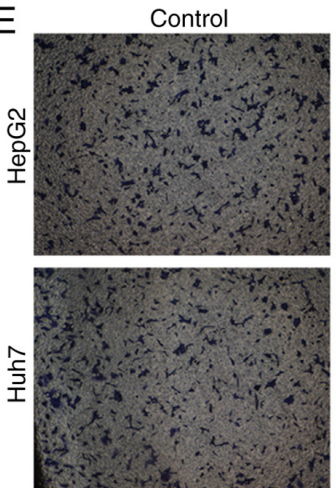
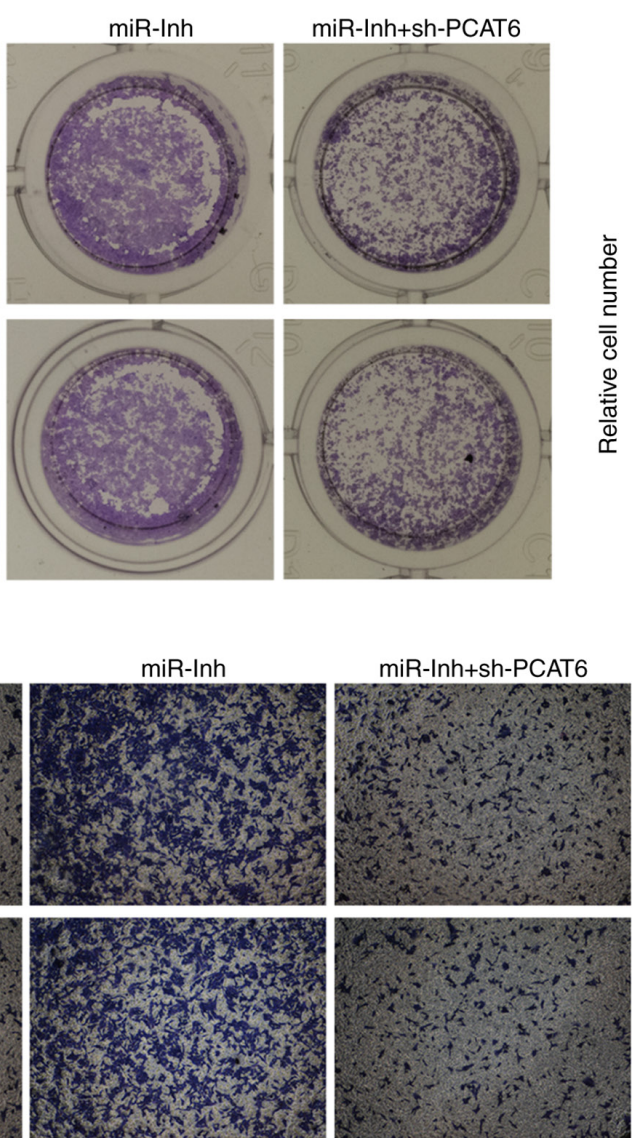
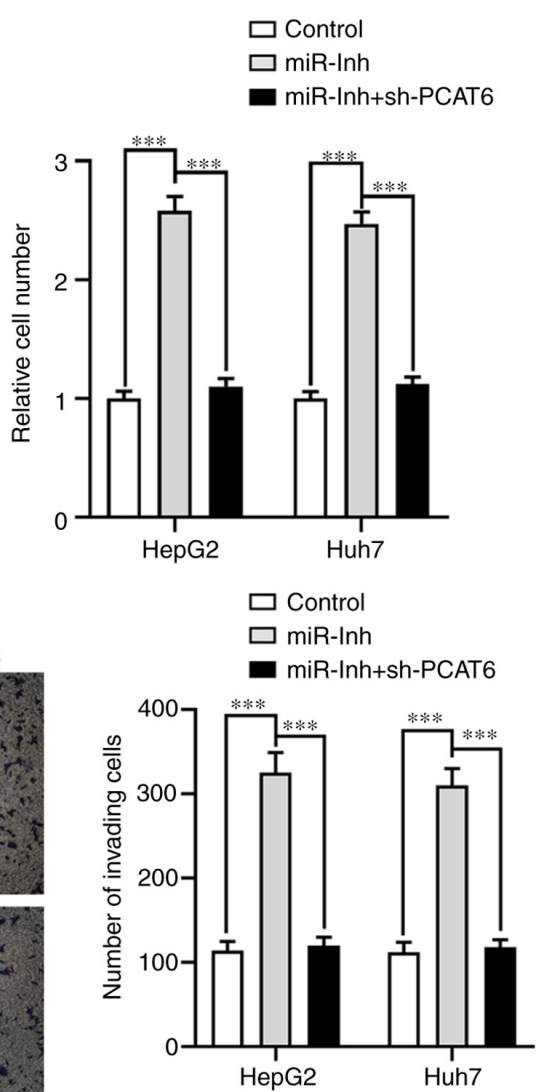

Figure 4. Inhibition of miR-326 reverses the inhibitory effect of PCAT6-knockdown on liver cancer cell proliferation and invasion ability of both HepG2 and Huh7 cells. (A) Reverse transcription-quantitative PCR was used to detect the expression of miR-326 in both HepG2 and Huh7 cells. (B and C) Cell Counting Kit-8 assay was used to measure the cell proliferation in both HepG2 and Huh7 cells after transfection with miR-Inh and sh-PCAT6. (D) After transfection, HepG2 and Huh7 cell colony formation abilities were determined. (E) Transwell assays were applied to detect the invasive activity of HepG2 and Huh7 cells after transfection with miR-Inh and sh-PCAT6. ${ }^{*} \mathrm{P}<0.05,{ }^{* *} \mathrm{P}<0.01$ and ${ }^{* * *} \mathrm{P}<0.001$ compared with control. miR-, microRNA; PCAT6, prostate cancer-associated transcript 6; miR-Inh, miR-326 inhibitor; sh-, short hairpin.

showed that inhibition of miR-326 significantly promoted the proliferation ability of both HepG2 and Huh7 cells compared with the control group ( $\mathrm{P}<0.05$; Fig. $4 \mathrm{~B}$ and $\mathrm{C})$. On the other hand, knockdown of PCAT6 reversed this effect. Similarly, the clone formation of both HepG2 and Huh7 cells when transfected with miR-Inh was markedly increased compared with the control group $(\mathrm{P}<0.001$; Fig. $4 \mathrm{D})$, while the number of liver cancer cell colonies were reduced by knocking down both miR-326 and PCAT6. Moreover, an elevated invasive ability of both HepG2 and Huh7 cells was observed when miR-326 was suppressed, which was abrogated by inhibition of PCAT6 ( $\mathrm{P}<0.001$; Fig. 4E). These results demonstrated that
PCAT6-silencing inhibited cell proliferation and invasion by upregulating miR-326 in liver cancer.

hnRNPA2B1 is a target of miR-326 in liver cancer cells. Next, the target of miR-326 was investigated using Starbase, which predicted that hnRNPA2B1 was a target gene (Fig. 3F). Considering the critical roles of hnRNPA2B1 in a number of malignant tumors (28), hnRNPA2B1 was selected as the target of miR-326. Notably, the GEPIA database showed that the mRNA level of hnRNPA2B1 was upregulated in liver cancer tissues compared with normal tissues $(\mathrm{P}<0.05$; Fig. $3 \mathrm{H})$. Moreover, the elevated expression of hnRNPA2B1 
in patients with liver cancer resulted in a shorter overall survival rate $(\mathrm{P}<0.01$; Fig. 3I). Likewise, the RT-qPCR analysis showed that the mRNA expression of hnRNPA2B1 in the HCCT group was higher compared with that in the ANT group $(\mathrm{P}<0.001$; Fig. 3J). In addition, Pearson's correlation analysis revealed that the mRNA expression of hnRNPA2B1 was negatively correlated with miR-326 expression in $\mathrm{HCC}$ tumor tissues $(\mathrm{P}<0.001$; Fig. $3 \mathrm{~K})$, while a positive correlation between hnRNPA2B1 and PCAT6 in liver cancer tissues was observed $(\mathrm{P}<0.001$; Fig. $3 \mathrm{~L})$. To further investigate this correlation, the luciferase reporter assay showed that miR-326 mimics significantly reduced the luciferase activity of WT-hnRNPA2B1 3'UTR, while this inhibition was blocked by mutation of the potential binding domains $(\mathrm{P}<0.001$; Fig. 3M). Western blotting showed that the protein level of hnRNPA2B1 was significantly downregulated in both HepG2 and Huh7 cells after transfection with the miR-326 mimic $(\mathrm{P}<0.001$; Figs. $3 \mathrm{G}$ and $\mathrm{S} 1 \mathrm{~A})$. Overall, these results suggested that miR-326 targeted and inhibited the expression of hnRNPA2B1.

PCAT6 promotes the proliferation and invasion of liver cancer cells via the miR-326/hnRNPA2B1 axis. Whether PCAT6 exhibited an antitumor effect on liver cancer cells via regulation of the miR-326/hnRNPA2B1 axis was further investigated. As shown in Figs. 5A and S1B-E, the protein levels of hnRNPA2B1 were significantly reduced in both HepG2 and Huh7 cells after transfection with sh-hnRNPA2B1 $(\mathrm{P}<0.001)$, whereas its expression was upregulated by inhibition of miR-326 or upregulation of PCAT6 in hnRNPA2B1-silenced liver cancer cells. Silencing of hnRNPA2B1 significantly decreased the proliferation $(\mathrm{P}<0.05$; Fig. $5 \mathrm{~B}$ and $\mathrm{C})$ and clone formation abilities compared with the control in both HepG2 and Huh7 cells ( $\mathrm{P}<0.001$; Fig. 5D and E). However, the inhibitory effect of hnRNPA2B1-silencing on liver cancer cell biological behavior was reversed by the combined downregulation of miR-326 and hnRNPA2B1 or PCAT6-overexpression and hnRNPA2B1-silencing $(\mathrm{P}<0.001$, Fig. 5B-E). Moreover, the invasion capacity of HepG2 and Huh7 cells was inhibited by knockdown of hnRNPA2B1 ( $\mathrm{P}<0.001$; Fig. 5F and G), an effect that could be attenuated through the inhibition of miR-326 or upregulation of PCAT6. Taken together, PCAT6-inhibition contributed to decreased cell proliferation and invasion of liver cancer cells via downregulation of hnRNPA2B1 and upregulation of miR-326.

Knockdown of PCAT6 suppresses tumor growth of liver cancer in vivo. Based on the antitumor effect of PCAT6-inhibition by regulating the miR-326/hnRNPA2B1 axis in vitro, the role of PCAT6 in vivo was further examined using a subcutaneous xenograft model. As shown in Fig. 6A and C, knockdown of PCAT6 significantly decreased tumor growth and tumor weight $(\mathrm{P}<0.001)$ compared with the sh-NC group at 28 days. Tumor volume in the sh-PCAT6 group was significantly lower compared with in the sh-NC group $(\mathrm{P}<0.05$, Fig. 6B). Moreover, RT-qPCR was performed to detect the expression of miR-326 and hnRNPA2B1 in tumor tissues derived from the xenograft mice model. PCAT6-silencing was found to enhance the expression of miR-326 while it reduced hnRNPA2B1 expression compared with the sh-NC group $(\mathrm{P}<0.001$, Fig. $6 \mathrm{D}$ and $\mathrm{E})$.
Therefore, knockdown of PCAT6 inhibited tumor growth by upregulating the inhibitory effect of miR-326 on hnRNPA2B1 expression in liver cancer.

\section{Discussion}

Despite advances and progress in the field of liver cancer research, liver cancer remains a worrisome burden worldwide and the number of deaths due to liver cancer in China increased by $33.80 \%$ between in 1990-2015 (29-31). In the present study, PCAT6 was upregulated in liver cancer tissues and associated with poor outcomes in patients. Moreover, loss-of-function experiments revealed that the knockdown of PCAT6 suppressed the proliferation and invasion capacities of liver cancer cells in vitro and in vivo. Mechanistically, inhibition of PCAT6 exerted an antitumor function in liver cancer by targeting miR-326 and downregulating hnRNPA2B1 expression.

Studies have confirmed that lncRNA is a useful biomarker for the early diagnosis and prognosis of numerous diseases including cancer $(32,33)$. For example, Sun et al (34) found five differentially expressed lncRNAs that are associated with shorter survival time in The Cancer Genome Atlas. Cai et al (35) reported that a nine lncRNA signature acts as a biomarker to predict overall survival in gastric cancer. Wan et al (36) demonstrated that serum PCAT6 could be used as a diagnostic and prognostic biomarker in patients with non-small-cell lung cancer. Moreover, lncRNAs may function as an oncogene or tumor suppressor gene involved in the progression of the malignant tumor by regulating the proliferation, invasion and migration of cancer cells $(6,37)$. For instance, Wang et al (38) demonstrated that a novel lncRNA MCM3AP antisense RNA 1 facilitates tumor growth of HCC by promoting HCC cell proliferation and reducing apoptosis. In the present study, PCAT6 was shown to be overexpressed in liver cancer tissues and cell lines, and inhibition of PCAT6 significantly decreased liver cancer cell proliferation and invasion. Of note, a recent study reported that PCAT6 serves as an oncogene to promote the development of non-small-cell lung cancer by binding to the enhancer of zeste homolog 2 (EZH2) and suppressing the expression of large tumor suppressor homolog 2 (39). Lv et al (40) showed that PCAT6-overexpression promotes cell proliferation, migration, invasion and reduces apoptosis through the activation of the $\mathrm{Wnt} / \beta$-catenin pathway in cervical cancer. Other studies have demonstrated that knockdown of PCAT6 reverses chemoresistance of cancer cells to 5-fluorouracil and enhances radiosensitivity in breast cancer cells $(41,42)$. The aforementioned results of the present study indicated that the oncogene PCAT6 might be an effective therapeutic target for patients with liver cancer.

Numerous studies have confirmed that lncRNA plays a major role in regulating cancer progression by targeting miRNA to regulate mRNA expression (43). For example, Dong et al (44) reported that silencing of PCAT6 restrains cell proliferation and epithelial-mesenchymal transition of gastric cancer cells by targeting miR-15a. Huang et al (16) confirmed that PCAT6 suppresses apoptosis and contributes to cell proliferation by upregulating anti-apoptotic protein apoptosis repressor 
A
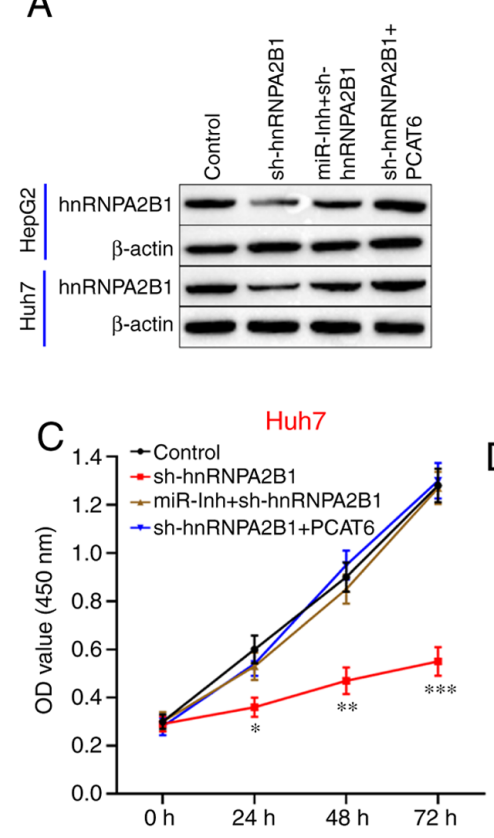

$\mathrm{F}$
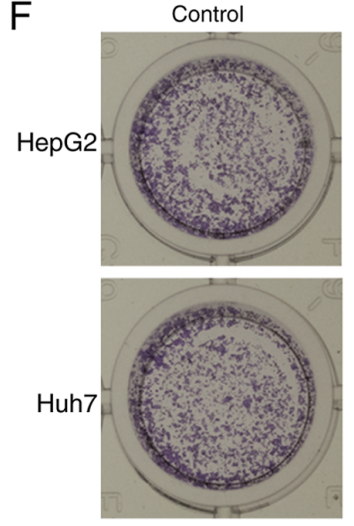

G

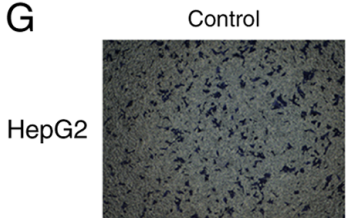

Huh7

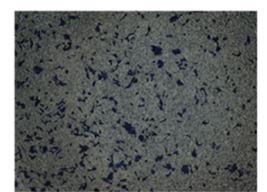

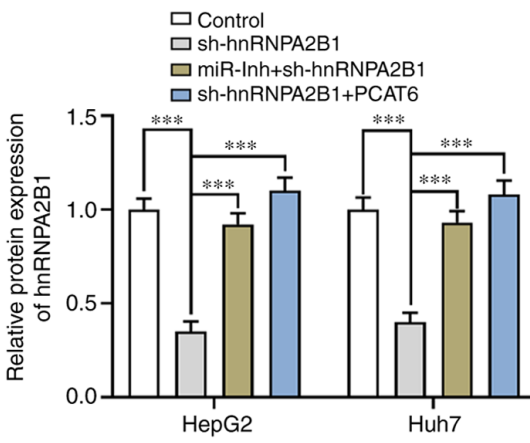

B
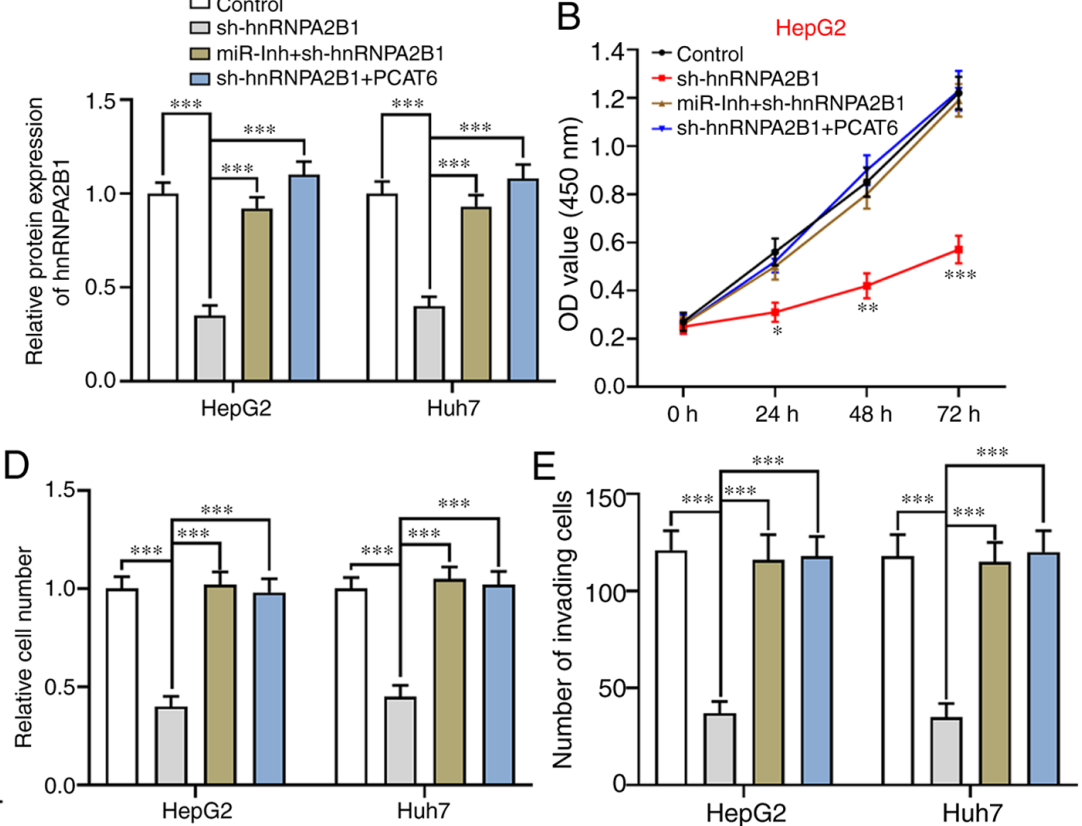

sh-hnRNPA2B1
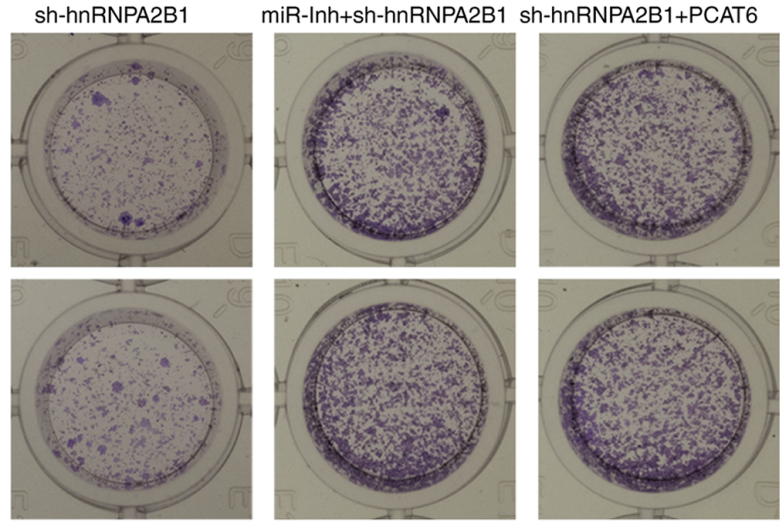

sh-hnRNPA2B1
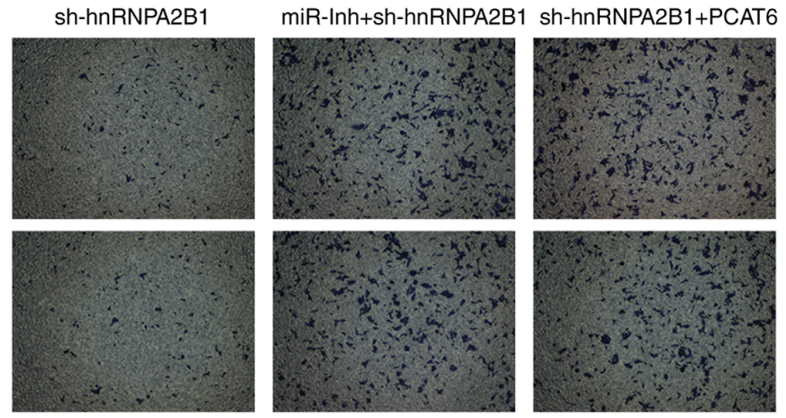

Figure 5. PCAT6 contributes to liver cancer cell proliferation and invasion by regulating the miR-326/hnRNPA2B1 axis. (A) Western blotting was used to detect the protein level of hnRNPA2B1 in both liver cancer cell after transfection with hnRNPA2B1 shRNA (sh-hnRNPA2B1), combined hnRNPA2B1 shRNA and miR-326 inhibitor (sh-hnRNPA2B1+miR-Inh), combined hnRNPA2B1 shRNA and pcDNA3.1-PCAT6 (sh-hnRNPA2B1+PCAT6). (B and C) After transfection, the proliferation ability of both HepG2 and Huh7 cells were determined by using a Cell Counting Kit-8 assay and (D and E) colony formation assay. (F and G) Cell invasion was detected using a Transwell assay. ${ }^{*} \mathrm{P}<0.05,{ }^{* *} \mathrm{P}<0.01$ and ${ }^{* * *} \mathrm{P}<0.001$. PCAT6, prostate cancer-associated transcript 6; miR-, microRNA; hnRNPA2B1, heterogeneous nuclear ribonucleoprotein A2B1; sh-, short hairpin-; miR-Inh, miR-326 inhibitor.

with caspase recruitment domain via EZH2 in colon cancer. Zhu et al (17) reported that PCAT6 promotes tumor progression in osteosarcoma by regulating the miR-185-5p/TGFBR1/2 axis. The results of the present study showed that PCAT6 targeted miR-326 to promote tumorigenesis of liver cancer by upregulating the expression of hnRNPA2B1. Notably, a number of studies have demonstrated that miR-326 acts as a tumor suppressor in cancer progression $(45,46)$. Lu et al $(47)$ reported that miR-326 is a novel
miRNA signature for HCC diagnosis and prognosis. Meanwhile, evidence suggests that miR-326 is involved in malignant phenotypes by targeting downstream genes or signaling pathways. For instance, overexpression of miR-326 targets LIM and SH3 protein 1 to suppress HCC cell proliferation and invasion and promote apoptosis (48). Mo et al (49) demonstrated that miR-326 inhibits HCC tumor growth by blocking the Akt/c-Myc pathway through targeting of 3-phosphoinositide-dependent protein 

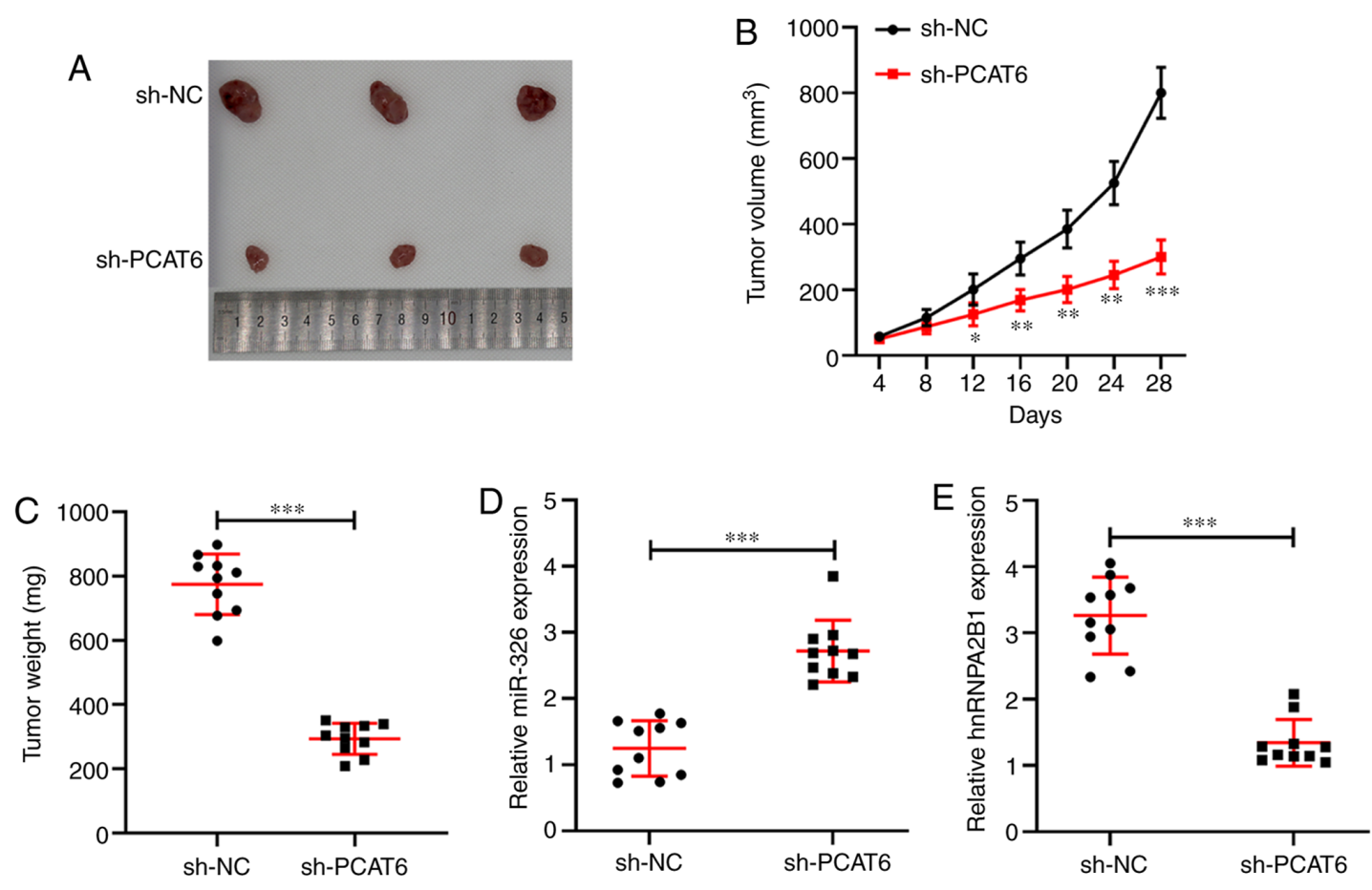

Figure 6. Inhibition of PCAT6 reduces tumor growth of liver cancer in vivo. (A) Tumor images from the two groups of nude mice. (B) Tumor volume was measured every four days. (C) Tumor weight was detected in each group. (D and E) Reverse transcription-quantitative PCR was performed to examine the expression of miR-326 and hnRNPA2B1 in tumor tissues derived from xenograft nude mice. ${ }^{*} \mathrm{P}<0.05,{ }^{* *} \mathrm{P}<0.01$ and ${ }^{* * *} \mathrm{P}<0.001$ compared with sh-NC. PCAT6, prostate cancer-associated transcript 6; miR-, microRNA; hnRNPA2B1, heterogeneous nuclear ribonucleoprotein A2B1; NC, negative control; sh-, short hairpin.

kinase 1. Upregulation of miR-326 reduces chemoresistance in numerous solid tumors, such as HCC (50), gastric cancer (51) and lung adenocarcinoma (52). In the present study, miR-326 directly targeted hnRNPA2B1, which is reported as an oncogene in HCC (53). In the present study, knockdown of hnRNPA2B1 significantly suppressed liver cancer cell proliferation and invasion, which is also in agreement with a previous study by Wang et al (54). Furthermore, previous studies demonstrated that silencing of hnRNPA2B1 inhibits malignant capability and induces apoptosis through downregulation of lin28B and inactivation of the PI3K/Akt pathway in tumors $(55,56)$. Notably, the data from the present study showed that inhibition of miR-326 or upregulation of PCAT6 significantly alleviated the inhibitory effect of hnRNPA2B1 deletion on the proliferation and invasion of liver cancer cells.

It was concluded that PCAT6 is upregulated in liver cancer tissues and significantly associated with poor prognosis in patients with liver cancer. Loss-of-function experiments demonstrated that knockdown of PCAT6 exhibited an anti-tumorigenic effect in liver cancer by downregulating hnRNPA2B1 expression through the targeting of miR-326. The study provided a theoretical basis for PCAT6 as a new diagnostic, prognostic and therapeutic target for patients with liver cancer.

\section{Acknowledgements}

Not applicable.

\section{Funding}

This work was supported by The Medicine and Health Discipline Platform Project of Zhejiang Province (grant no. 2018RC019) and The Medicine and Health Science Project of Zhejiang Province (grant no. 2020KY483).

\section{Availability of data and materials}

The datasets used and/or analyzed in the current study are available from the corresponding author on request.

\section{Authors' contributions}

JL and GS designed the experiments. JL, JZ and WH performed experiments. WH, $\mathrm{HZ}$ and $\mathrm{ZZ}$ analyzed the data. $\mathrm{JZ}$ and WH confirmed the authenticity of all raw data. JL and JZ drafted the manuscript. GS revised manuscript. All authors read and approved the final manuscript.

\section{Ethics approval and consent to participate}

The study was approved by The Ethics Committee of Zhejiang Cancer Hospital (Hangzhou, China; approval no. IRB-2020-6). All patients have signed written informed consent. The animal study was approved by the Animal Care and Use Committee of the Zhejiang Cancer Hospital (approval no. IRB-2019-5).

\section{Patient consent for publication}

Not applicable.

\section{Competing interests}

The authors declare that they have no competing interests. 


\section{References}

1. Global Burden of Disease Liver Cancer Collaboration, Akinyemiju T, Abera S, Ahmed M, Alam N, Alemayohu MA, Allen C, Al-Raddadi R, Alvis-Guzman N, Amoako Y, et al: The burden of primary liver cancer and underlying etiologies from 1990 to 2015 at the global, regional, and national level: Results from the global burden of disease study 2015. JAMA Oncol 3 : 1683-1691, 2017.

2. Mavros MN, Mayo SC, Hyder O and Pawlik TM: A systematic review: Treatment and prognosis of patients with fibrolamellar hepatocellular carcinoma. J Am Coll Surg 215: 820-830, 2012.

3. Tan N, Zhu B, Shu H, Tao YF, Wu JR, Fang M, Li CR, Chen ZQ and Ou C: Effect of lncRNA-BC200 on proliferation and migration of liver cancer cells in vitro and in vivo. Oncol Rep 43 461-470, 2020

4. Stipa F, Yoon SS, Liau KH, Fong Y, Jarnagin WR, D'Angelica M, Abou-Alfa G, Blumgart LH and DeMatteo RP: Outcome of patients with fibrolamellar hepatocellular carcinoma. Cancer 106 : 1331-1338, 2006.

5. Kakar S, Burgart LJ, Batts KP, Garcia J, Jain D and Ferrell LD Clinicopathologic features and survival in fibrolamellar carcinoma: Comparison with conventional hepatocellular carcinoma with and without cirrhosis. Mod Pathol 18: 1417-1423, 2005.

6. Bhan A, Soleimani M and Mandal SS: Long noncoding RNA and cancer: A new paradigm. Cancer Res 77: 3965-3981, 2017.

7. Arun G, Diermeier SD and Spector DL: Therapeutic targeting of long non-coding RNAs in cancer. Trends Mol Med 24: 257-277, 2018.

8. Liu Y, Feng W, Liu W, Kong X, Li L, He J, Wang D, Zhang M, Zhou G, Xu W, et al: Circulating lncRNA ABHD11-AS1 serves as a biomarker for early pancreatic cancer diagnosis. J Cancer 10: 3746-3756, 2019

9. Chi Y, Wang D, Wang J, Yu W and Yang J: Long non-coding RNA in the pathogenesis of cancers. Cells 8: 1015, 2019.

10. Zhong G, Wang K, Li J, Xiao S, Wei W and Liu J: Determination of serum exosomal H19 as a noninvasive biomarker for breast cancer diagnosis. Onco Targets Ther 13: 2563-2571, 2020.

11. Xu W, Zhou G, Wang H, Liu Y, Chen B, Chen W, Lin C, Wu S Gong A and Xu M: Circulating lncRNA SNHG11 as a nove biomarker for early diagnosis and prognosis of colorectal cancer. Int J Cancer 146: 2901-2912, 2020.

12. Zhang G, Chi N, Lu Q, Zhu D and Zhuang Y: LncRNA PTCSC3 is a biomarker for the treatment and prognosis of gastric cancer. Cancer Biother Radiopharm 35: 77-81, 2020

13. Yao Z, Zhang Y, Xu D, Zhou X, Peng P, Pan Z, Xiao N, Yao J and Li Z: Research progress on long non-coding RNA and radiotherapy. Med Sci Monit 25: 5757-5770, 2019.

14. Wan L, Zhang L, Fan K, Cheng ZX, Sun QC and Wang JJ Knockdown of long noncoding RNA PCAT6 inhibits proliferation and invasion in lung cancer cells. Oncol Res 24: 161-170, 2016.

15. Bai F, Zhang N, Fang W, He X, Zheng Y and Gu D: PCAT6 mediates cellular biological functions in gastrointestinal stromal tumor via upregulation of PRDX5 and activation of Wnt pathway. Mol Carcinog 59: 661-669, 2020.

16. Huang W, Su G, Huang X, Zou A, Wu J, Yang Y, Zhu Y, Liang S, Li D, Ma F and Guo L: Long noncoding RNA PCAT6 inhibits colon cancer cell apoptosis by regulating anti-apoptotic protein ARC expression via EZH2. Cell Cycle 18: 69-83, 2019.

17. Zhu C, Huang L, Xu F, Li P, Li P and Hu F: LncRNA PCAT6 promotes tumor progression in osteosarcoma via activation of TGF- $\beta$ pathway by sponging miR-185-5p. Biochem Biophys Res Commun 521: 463-470, 2020.

18. Tang X, Feng D, Li M, Zhou J, Li X, Zhao D, Hao B, Li D and Ding K: Transcriptomic analysis of mRNA-lncRNA-miRNA interactions in hepatocellular carcinoma. Sci Rep 9: 16096, 2019.

19. Gnoni A, Santini D, Scartozzi M, Russo A, Licchetta A, Palmieri V, Lupo L, Faloppi L, Palasciano G, Memeo V, et al: Hepatocellular carcinoma treatment over sorafenib: Epigenetics, microRNAs and microenvironment. Is there a light at the end of the tunnel? Expert Opin Ther Targets 19: 1623-1635, 2015.

20. He S, Yang J, Jiang S, Li Y and Han X: Circular RNA circ_0000517 regulates hepatocellular carcinoma development via miR-326/IGF1R axis. Cancer Cell Int 20: 404, 2020.

21. Council for International Organizations of Medical Sciences (CIOMS): International Ethical Guidelines for Biomedical Research Involving Human Subjects. CIOMS, Geneva, p60, 2002.
22. Tang Z, Li C, Kang B, Gao G, Li C and Zhang Z: GEPIA: A web server for cancer and normal gene expression profiling and interactive analyses. Nucleic Acids Res 45: W98-W102, 2017.

23. Zheng J, Luo J, Zeng H, Guo L and Shao G: ${ }^{125}$ I suppressed the Warburg effect via regulating miR-338/PFKL axis in hepatocellular carcinoma. Biomed Pharmacother 119: 109402, 2019.

24. Livak KJ and Schmittgen TD: Analysis of relative gene expression data using real-time quantitative PCR and the 2(-Delta Delta C(T)) method. Methods 25: 402-408, 2001

25. Zeng H, Zheng J, Wen S, Luo J, Shao G and Zhang Y: MicroRNA-339 inhibits human hepatocellular carcinoma proliferation and invasion via targeting ZNF689. Drug Des Devel Ther 13: 435-445, 2019

26. Li JH, Liu S, Zhou H, Qu LH and Yang JH: starBase v2.0: Decoding miRNA-ceRNA, miRNA-ncRNA and protein-RNA interaction networks from large-scale CLIP-Seq data. Nucleic Acids Res 42: D92-D97, 2014.

27. Li HW and Liu J: Circ 0009910 promotes proliferation and metastasis of hepatocellular carcinoma cells through miR-335-5p/ROCK1 axis. Eur Rev Med Pharmacol Sci 24: $1725-1735,2020$

28. Liu Y and Shi SL: The roles of hnRNP A2/B1 in RNA biology and disease. Wiley Interdiscip Rev RNA 12: e1612, 2021.

29. Chen W, Zheng R, Baade PD, Zhang S, Zeng H, Bray F, Jemal A, $\mathrm{Yu}$ XQ and He J: Cancer statistics in China, 2015. CA Cancer J Clin 66: 115-132, 2016.

30. Chen J, Zhang Y, Chen Y and Ding L: Incidence trend of liver cancer: An analysis of 40 years data from Qidong population-based cancer registry. Zhongguo Zhong Liu 23: 621-628, 2014

31. Sun Z, Chen T, Thorgeirsson SS, Zhan Q, Chen J, Park JH, Lu P, Hsia CC, Wang N, Xu L, et al: Dramatic reduction of liver cancer incidence in young adults: 28 year follow-up of etiological interventions in an endemic area of China. Carcinogenesis 34 1800-1805, 2013

32. Kondo Y, Shinjo K and Katsushima K: Long non-coding RNAs as an epigenetic regulator in human cancers. Cancer Sci 108 1927-1933, 2017

33. Camacho CV, Choudhari R and Gadad SS: Long noncoding RNAs and cancer, an overview. Steroids 133: 93-95, 2018.

34. Sun Y, Zhang F, Wang L, Song X, Jing J, Zhang F, Yu S and Liu H: A five lncRNA signature for prognosis prediction in hepatocellular carcinoma. Mol Med Rep 19: 5237-5250, 2019.

35. Cai C, Yang L, Tang Y, Wang H, He Y, Jiang H and Zhou K: Prediction of overall survival in gastric cancer using a nine-lncRNA. DNA Cell Biol 38: 1005-1012, 2019.

36. Wan L, Zhang L, Fan K and Wang JJ: Diagnostic significance of circulating long noncoding RNA PCAT6 in patients with non-small cell lung cancer. Onco Targets Ther 10: 5695-5702, 2017.

37. Peng WX, Koirala P and Mo YY: LncRNA-mediated regulation of cell signaling in cancer. Oncogene 36: 5661-5667, 2017.

38. Wang Y, Yang L, Chen T, Liu X, Guo Y, Zhu Q, Tong X, Yang W, $\mathrm{Xu}$ Q, Huang D and Tu K: A novel IncRNA MCM3AP-AS1 promotes the growth of hepatocellular carcinoma by targeting miR-194-5p/FOXA1 axis. Mol Cancer 18: 28, 2019.

39. Shi X, Liu Z, Liu Z, Feng X, Hua F, Hu X, Wang B, Lu K and Nie F: Long noncoding RNA PCAT6 functions as an oncogene by binding to EZH2 and suppressing LATS2 in non-small-cell lung cancer. EBioMedicine 37: 177-187, 2018.

40. Lv XJ, Tang Q, Tu YQ, Yan DD and Wei QC: Long noncoding RNA PCAT6 regulates cell growth and metastasis via Wnt/ $\beta$-catenin pathway and is a prognosis marker in cervical cancer. Eur Rev Med Pharmacol Sci 23: 1947-1956, 2019.

41. Wu H, Zou Q, He H, Liang Y, Lei M, Zhou Q, Fan D and Shen L: Long non-coding RNA PCAT6 targets miR-204 to modulate the chemoresistance of colorectal cancer cells to 5-fluorouracil-based treatment through HMGA2 signaling. Cancer Med 8: 2484-2495, 2019.

42. Shi R, Wu P, Liu M, Chen B and Cong L: Knockdown of lncRNA PCAT6 enhances radiosensitivity in triple-negative breast cancer cells by regulating miR-185-5p/TPD52 axis. Onco Targets Ther 13: 3025-3037, 2020

43. Lin C, Yuan G, Hu Z, Zeng Y, Qiu X, Yu H and He S: Bioinformatics analysis of the interactions among lncRNA, miRNA and mRNA expression, genetic mutations and epigenetic modifications in hepatocellular carcinoma. Mol Med Rep 19: 1356-1364, 2019

44. Dong D, Lun Y, Sun B, Sun H, Wang Q, Yuan G and Quan J: Silencing of long non-coding RNA PCAT6 restrains gastric cancer cell proliferation and epithelial-mesenchymal transition by targeting microRNA-15a. Gen Physiol Biophys 39: 1-12, 2020. 
45. Zou X, Zhong J, Li J, Su Z, Chen Y, Deng W, Li Y, Lu S, Lin Y, Luo L, et al: miR-362-3p targets nemo-like kinase and functions as a tumor suppressor in renal cancer cells. Mol Med Rep 13: 994-1002, 2016.

46. Wang D, Wang H, Li Y and Li Q: MiR-362-3p functions as a tumor suppressor through targeting MCM5 in cervical adenocarcinoma. Biosci Rep 38: BSR20180668, 2018.

47. Lu M, Kong X, Wang H, Huang G, Ye C and He Z: A novel microRNAs expression signature for hepatocellular carcinoma diagnosis and prognosis. Oncotarget 8: 8775-8784, 2017.

48. Hu S, Ran Y, Chen W, Zhang Y and Xu Y: MicroRNA-326 inhibits cell proliferation and invasion, activating apoptosis in hepatocellular carcinoma by directly targeting LIM and SH3 protein 1. Oncol Rep 38: 1569-1578, 2017.

49. Mo Y, He L, Lai Z, Wan Z, Chen Q, Pan S, Li L, Li D, Huang J Xue F and Che S: Gold nano-particles (AuNPs) carrying miR-326 targets PDK1/AKT/c-myc axis in hepatocellular carcinoma. Artif Cells Nanomed Biotechnol 47: 2830-2837, 2019.

50. Ma J, Wang T, Guo R, Yang X, Yin J, Yu J, Xiang Q, Pan X, Tang $H$ and Lei $X$ : Involvement of miR-133a and miR-326 in ADM resistance of HepG2 through modulating expression of ABCC1. J Drug Target 23: 519-524, 2015.

51. Song W, Qian Y, Zhang MH, Wang H, Wen X, Yang XZ and Dai WJ: The long non-coding RNA DDX11-AS1 facilitates cell progression and oxaliplatin resistance via regulating miR-326/IRS1 axis in gastric cancer. Eur Rev Med Pharmacol Sci 24: 3049-3061, 2020.
52. Yu W, Peng W, Sha H and Li J: Hsa circ 0003998 promotes chemoresistance via modulation of $\mathrm{mi} \overline{\mathrm{R}}-32 \overline{6}$ in lung adenocarcinoma cells. Oncol Res 27: 623-628, 2019.

53. Mizuno H, Honda M, Shirasaki T, Yamashita T, Yamashita $\mathrm{T}$, Mizukoshi E and Kaneko S: Heterogeneous nuclear ribonucleoprotein A2/B1 in association with hTERT is a potential biomarker for hepatocellular carcinoma. Liver Int 32: 1146-1155, 2012.

54. Wang H, Liang L, Dong Q, Huan L, He J, Li B, Yang C, Jin H, Wei L, Yu C, et al: Long noncoding RNA miR503HG, a prognostic indicator, inhibits tumor metastasis by regulating the HNRNPA2B1/NF- $\kappa$ B pathway in hepatocellular carcinoma. Theranostics 8: 2814-2829, 2018.

55. Yang Y, Wei Q, Tang Y, Wang Y, Luo Q, Zhao H, He M, Wang H, Zeng Q, Lu W, et al: Loss of hnRNPA2B1 inhibits malignant capability and promotes apoptosis via down-regulating Lin28B expression in ovarian cancer. Cancer Lett 475: 43-52, 2020.

56. Shi X, Ran L, Liu Y, Zhong SH, Zhou PP, Liao MX and Fang W: Knockdown of hnRNP A2/B1 inhibits cell proliferation, invasion and cell cycle triggering apoptosis in cervical cancer via PI3K/AKT signaling pathway. Oncol Rep 39: 939-950, 2018.

(i) $(5)$ This work is licensed under a Creative Commons Attribution-NonCommercial-NoDerivatives 4.0 International (CC BY-NC-ND 4.0) License. 